\title{
Comparação do LASER e do LED no processo de cicatrização em feridas cutâneas: uma revisão
}

\author{
Comparison of LASER and LED in the process of healing cutaneous wounds: a review
}

\author{
Simone de Paula \\ a Fisioterapeuta. Doutora em Saúde da Criança pela PUCRS. Especialista em Fisioterapia Dermatofuncional (COFFITO). \\ Docente do Curso de Fisioterapia da Universidade Feevale.
}

RESUMO Objetivo: O objetivo desta revisão foi apresentar e comparar as evidências científicas atuais sobre os efeitos terapêuticos do LASER e do LED de baixa potência no processo de cicatrização cutânea através da análise de estudos experimentais em roedores.

Materiais e Métodos: Revisão bibliográfica, realizada por meio de pesquisa nas bases de dados Medline/PubMed e SCiELO. Foram selecionados artigos originais e experimentais disponíveis na íntegra que objetivaram comparar os efeitos terapêuticos do LASER e do LED no processo de cicatrização cutânea. As palavras-chaves e os operadores boleanos utilizados para a busca nas bases de dados foram: wound healing OR healing AND laser AND led. Foram incluídos artigos publicados nos idiomas inglês e português.

Resultados: De um total de 232 artigos encontrados, cinco estudos foram selecionados para a presente revisão de literatura. Os anos de publicação variaram de 2007 a 2015. Três estudos utilizaram LASERs e LEDs com comprimentos de onda vermelhos $(\lambda<700 \mathrm{~nm}$ ) e dois estudos aplicaram ambos os espectros vermelhos e infra-vermelhos nos grupos analisados. A maioria dos estudos realizou um protocolo com, pelo menos, duas aplicações do LASER ou LED através da técnica de contato. A densidade de energia variou de $2,5 \mathrm{~J} / \mathrm{cm}^{2}$ a $40 \mathrm{~J} / \mathrm{cm}^{2}$.

Conclusão: Apesar da variabilidade metodológica apresentada nas investigações, os resultados destes estudos mostraram desfechos histológicos e imunohistoquímicos satisfatórios, sugerindo que estas terapias possam favorecer significativamente o processo de reparo tecidual. No entanto, estudos adicionais necessitam ser realizados a fim de definir estratégias clínicas seguras e efetivas para o uso da fototerapia na cicatrização de feridas.

Palavras-chave: cicatrização; fototerapia; LASER.

Objective: The objective of this review was to present and compare the current scientific evidence on the therapeutic effects of low level LASER and LED in the cutaneous healing process through the analysis of experimental studies in rodents.

Materials and Methods: Literature review, carried out through a search in the Medline/PubMed and SciELO databases. Original and experimental articles available in full that aimed to compare the therapeutic LASER and LED effects on the skin healing process were selected. The keywords and boolean operators used to search the databases were: wound healing OR healing AND laser AND led. Articles published in English and Portuguese were included.

Results: From a total of 232 articles found, five studies were selected for this literature review. Year of publication ranged from 2007 to 2015. Three studies used LASERs and LEDs with red wavelengths $(\lambda<700 \mathrm{~nm})$ and two studies used both red and infrared spectra in the analyzed groups. Most of the studies conducted a protocol with at least two applications of LASER or LED through the contact technique. The energy density ranged from $2.5 \mathrm{~J} / \mathrm{cm}^{2}$ to $40 \mathrm{~J} / \mathrm{cm}^{2}$.

Conclusion: Despite the methodological variability in the researchers found, the results of these studies showed satisfactory histological and immunohistochemical outcomes, suggesting that these therapies can significantly facilitate the tissue repair process. However, further studies should be performed in order to establish safe and effective clinical strategies for the use of phototherapy in wound healing.

Keywords: wound healing; phototherapy; LASER. 


\section{INTRODUÇÃO}

A cicatrização cutânea é um processo fisiológico dinâmico e complexo, caracterizado por uma grande variedade de eventos celulares, moleculares e bioquímicos que interagem para que ocorra a reconstituição tecidual. Logo após o surgimento de uma lesão, há uma complexa liberação de mediadores que dão início ao processo de reparo, o qual se inicia pela inflamação, fase da migração de leucócitos e plaquetas; seguido pela fase proliferativa ou granulação, com destaque para a angiogênese e para o aumento no número de fibroblastos; e finalmente, concluise com a remodelação. Esta última fase dura meses e tem como meta a melhoria nos componentes das fibras colágenas e a reabsorção de água para aumentar a força da cicatriz e diminuir sua espessura ${ }^{1,2}$. Em virtude da complexidade do processo de reparo tecidual, inúmeros fatores exógenos e endógenos podem alterar os mecanismos de cicatrização da pele resultando em disfunções na integridade cutânea, tais como a fibrose excessiva ou a persistência de feridas ${ }^{1,3}$.

As feridas cutâneas constituem um problema de saúde pública no Brasil e no mundo. Estima-se que as dificuldades de cicatrização atinjam 1-2\% da população em geral destinando, assim, 2\% do orçamento em saúde para o tratamento de feridas ${ }^{4}$. Apenas nos Estados Unidos, as feridas crônicas afetam 6,5 milhões de pessoas resultando em quadros de dores crônicas, alterações emocionais, infecciosas, metabólicas e estéticas, além de limitações funcionais importantes, tais como alterações de marcha e dificuldades de deambulação ${ }^{5}$. Em virtude da significância clínica e sócio-econômica das feridas cutâneas, soluções inovadoras para melhorar a qualidade de vida dos pacientes acometidos por deficiências no processo de reparação tecidual têm sido investigadas, incluindo o uso de células-tronco, fatores de crescimento, medicamentos, oxigenação hiperbárica e a utilização de recursos da Fisioterapia, tais como o ultrassom e, mais recentemente, a fototerapia ${ }^{6-9}$.

A fototerapia, em seu amplo sentido, significa o uso terapêutico de qualquer tipo de luz. Atualmente, o conceito moderno de fototerapia se define como o uso de níveis baixos de energia da luz para promover efeitos atérmicos e não traumáticos nos tecidos. A primeira fonte de luz usada na Medicina foi a luz natural do sol, abordagem utilizada por mais de 1000 anos por povos na Índia, China, Grégia e Egito para tratar doenças de pele, entre as quais o vitiligo e a psoríase. Mais tarde, entre os séculos XVIII e XIX, os mecanismos de ação da radiação ultravioleta sobre os seres humanos passaram a ser esclarecidos, especialmente através de estudos do médico e cientista Niels Finsen, ganhador do prêmio Nobel de Medicina de 1903 por seus estudos sobre a fototerapia ultravioleta no lúpus vulgar ${ }^{9-11}$.

Já em meados da década de 60, as primeiras aplicações clínicas da fototerapia com LASER de baixa potência foram relatadas através dos estudos de Mester, um médico húngaro que descobriu, por acaso, o potencial fotobiológico do LASER de rubi ${ }^{12}$. Desde então, a laserterapia tem sido um dos recursos fototerapêuticos mais utilizados na prática clínica para reduzir a inflamação e aumentar o reparo de diferentes tecidos. Além disso, por ser considerada uma terapia não invasiva, indolor e não térmica, a luz monocromática e coerente emitida pelo LASER vem despertando crescente interesse dos pesquisadores quanto ao seu mecanismo de ação e a seus efeitos terapêuticos. Resultados positivos sobre o uso do LASER na faixa espectral do vermelho ao infravermelho (600-1200nm) têm sido demonstrados através de estudos experimentais e clínicos, indicando que esta modalidade terapêutica pode ter eficiente ação antiinflamatória e analgésica, além de aumentar a produção de adenosina trifosfato (ATP), facilitando a proliferação celular e a neoformação vascular e, consequentemente, promovendo qualidade e rapidez no processo cicatricial de feridas cutâneas ${ }^{13,14}$.

Mais recentemente o diodo emissor de luz conhecido como LED tem sido apresentado como uma abordagem alternativa ao alto custo da laserterapia. Diferentemente do LASER, a terapia com LEDs possui restrita base literária e distingue-se por emitir luzes policromáticas e não coerentes. No entanto, nos últimos anos a National Aeronautics and Space Administration (NASA) tem colaborado com diversas pesquisas sobre o uso do LED na reparação de ferimentos, demonstrando resultados seguros e promissores para o tratamento de diversas alterações dermatofuncionais ${ }^{15,16}$.

Apesar dos avanços científicos sobre o uso da luz no reparo cutâneo, aspectos como fluência, comprimento de onda, modo de aplicação e as diferenças relacionadas à coerência e à colimação da luz ainda necessitam ser esclarecidos para a otimização desta abordagem terapêutica no tratamento fisioterapêutico de feridas. Neste contexto, o objetivo desta revisão foi apresentar e comparar as evidências científicas atuais sobre os efeitos terapêuticos do LASER e do LED de baixa potência no processo de cicatrização cutânea através da análise de estudos comparativos e experimentais em roedores.

\section{MATERIAIS E MÉTODOS}

O estudo consiste em uma revisão bibliográfica, realizada por meio de pesquisa nas bases de dados Medline/PubMed e 
SciELO. Foram selecionados artigos originais e experimentais disponíveis na íntegra contendo análises comparativas sobre os efeitos terapêuticos do LASER e do LED no processo de cicatrização cutânea. As palavras-chaves e os operadores boleanos utilizados para a busca nas bases de dados foram: wound healing OR healing AND laser AND led. Foram incluídos artigos publicados nos idiomas inglês e português. Não foram utilizados filtros em relação ao ano de publicação dos estudos.

Os critérios de inclusão para a seleção dos ensaios experimentais desta revisão foram: 1) o uso de LASER e LED nos grupos experimentais; 2) a análise estatística comparativa entre o LASER e o LED nos grupos experimentais; 3) a presença desfechos histológicos, funcionais e/ou imunohistoquímicos relacionados à cicatrização de feridas cutâneas; 4) o comprimento de onda, a fluência e o modo de aplicação claramente definidos na metodologia; e 5) uso in vivo de camundongos ou ratos nos grupos experimentais. Foram excluídos os estudos in vitro ou com metodologia clínica, as revisões e metaanálises e os artigos envolvendo tecidos não cutâneos. A Figura 1 resume a estratégia de seleção dos artigos para esta revisão.

Após a análise dos títulos e resumos (abstracts) dos artigos selecionados através da pesquisa boleada, realizouse uma triagem dos artigos disponíveis na íntegra e que contemplavam os critérios de inclusão e exclusão. Os 5 (cinco) artigos selecionados foram dispostos em uma tabela para facilitar a visualização dos resultados. As principais variáveis analisadas entre os estudos foram: fluência, frequência e modo de aplicação, desfechos histológicos e imunohistoquímicos.

\section{RESULTADOS E DISCUSSÃO}

A busca na base de dados Medline/PubMed, Lilacs e SciELO resultou num total de 232 citações. Destas 232 citações somente cinco estudos experimentais $(n=5)$ em ratos Wistar preencheram adequadamente aos critérios de inclusão desta revisão. Os anos de publicação variaram de 2007 a 2015. Dos cinco estudos analisados, quatro foram publicados no periódico Lasers in Medical Science, com fator de impacto de 2.402, e um foi publicado no periódico Photomedicine and Laser Surgery, com fator de impacto de 1.634. Os 4 estudos avaliados nesta revisão são resumidos na Tabela 1 .

Para a indução do modelo de ferida cutânea três estudos realizaram um corte no dorso do animal com uma área de 0,5-1 cm $\mathrm{cm}^{2,17-19}$ e um realizou um corte de $15 \mathrm{~mm}$ de diâmetro na coxa do animal ${ }^{20}$. Dois estudos ${ }^{17,19}$ compararam a eficácia do LASER e do LED em modelos animais de doenças consideradas de risco para dificuldades de cicatrização

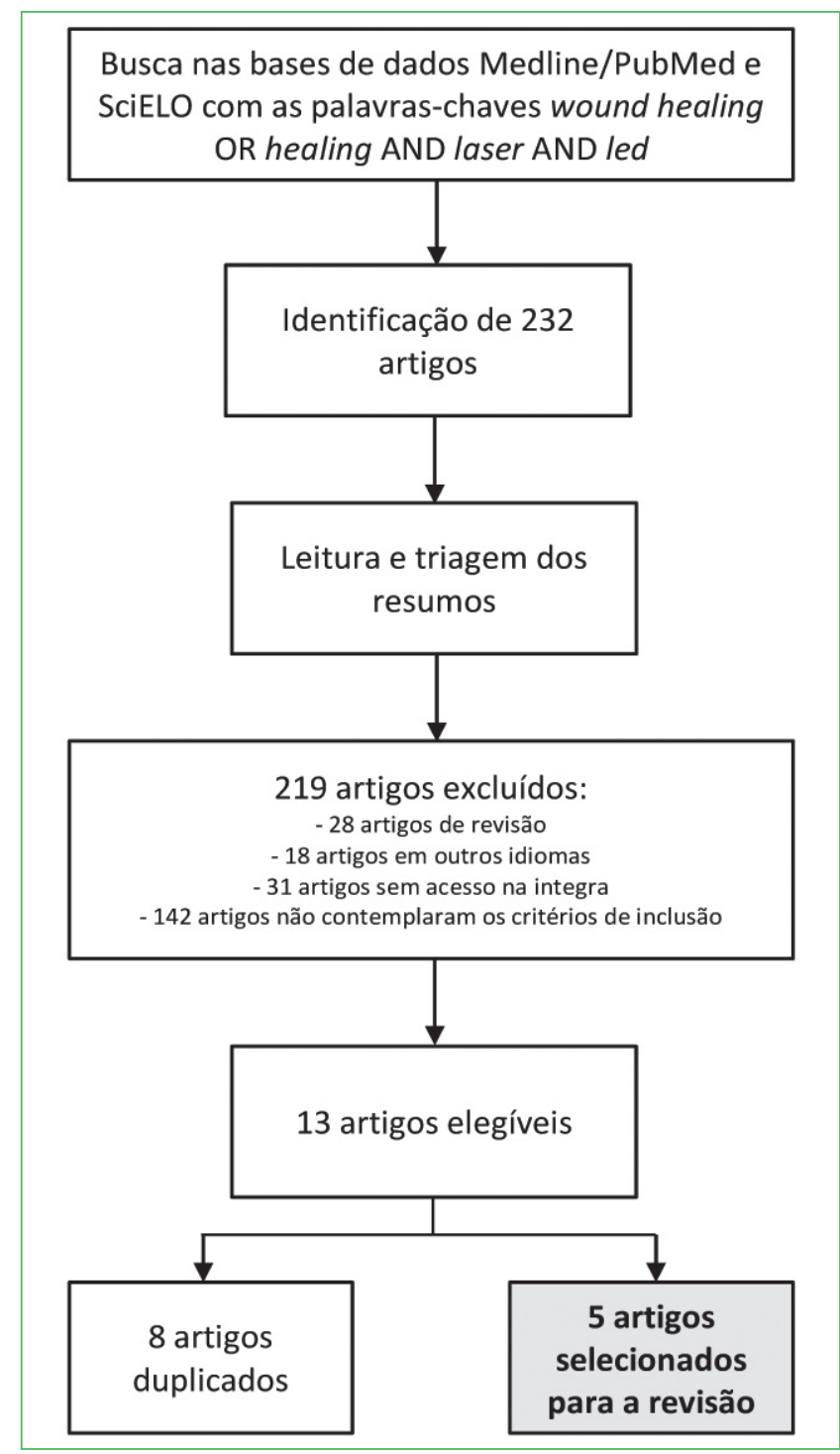

Figura 1. Fluxograma com a estratégia de seleção para a inclusão dos artigos.

cutânea (diabetes e deficiência de ferro, respectivamente). Dois estudos realizaram a pesquisa em animais saudáveis ${ }^{18,20}$ e uma pesquisa avaliou os efeitos da fototerapia em um modelo animal de queimadura ${ }^{21}$.

Em relação aos parâmetros fototerapêuticos escolhidos, três estudos ${ }^{17,19,20}$ utilizaram LASERs e LEDs com comprimentos de onda vermelhos $(\lambda<700 \mathrm{~nm})$. De Souza et al. ${ }^{18}$ e Catão et al. ${ }^{21}$ aplicaram ambos os espectros vermelhos e infra-vermelhos nos grupos analisados. A maioria dos estudos realizou um protocolo com, pelo menos, duas aplicações do LASER ou LED, exceto o trabalho de Dall Agnol et al. ${ }^{17}$ que utilizou uma dose única logo após o procedimento cirúrgico. A densidade de energia variou de $2,5 \mathrm{~J} / \mathrm{cm}^{2}$ a $40 \mathrm{~J} / \mathrm{cm}^{2}$ e a técnica de aplicação foi o método de contato com auxílio de papel filme, exceto no estudo de Dall Agnol et al. ${ }^{17}$ que aplicou o LASER ou LED a uma distância de $1 \mathrm{~cm}$ da ferida ${ }^{17}$. 
Tabela 1. Estudos experimentais sobre os efeitos do LASER e do LED no processo de cicatrização cutânea analisados nesta revisão.

\begin{tabular}{|c|c|c|c|c|c|}
\hline Estudo & Objetivo & $\begin{array}{l}\text { Fototerapia (fluência, } \\
\text { freqüência e modo) }\end{array}$ & Desfechos histológicos & $\begin{array}{c}\text { Desfechos } \\
\text { imunohistoquímicos }\end{array}$ & Conclusão \\
\hline Corazza et al. ${ }^{20}$ & $\begin{array}{l}\text { Comparar os efeitos } \\
\text { fotoindutores das } \\
\text { luzes coerentes e } \\
\text { não coerentes na } \\
\text { formação vascular e } \\
\text { no fechamento de } \\
\text { feridas cutâneas. }\end{array}$ & $\begin{array}{l}\text { LASER de diodo com } \\
\lambda 660 \mathrm{~nm} \text { e LED de } \\
\lambda 635 \mathrm{~nm} \text {. } \\
\text { O tratamento foi diário, } \\
\text { iniciado } 6 \mathrm{~h} \text { após o } \\
\text { procedimento cirúrgico } \\
\text { e aplicado em } 6 \text { pontos } \\
\text { ao redor da ferida. } \\
\text { A aplicação foi pontual } \\
\text { em contato com a pele } \\
\text { (a ferida foi forrada } \\
\text { com papel filme). }\end{array}$ & $\begin{array}{l}\text { A densidade de } \\
\text { vasos sanguíneos foi } \\
\text { significativamente } \\
\text { maior em todos os } \\
\text { animais tratados quando } \\
\text { comparados aos } \\
\text { controles nos dias } 3 \text { e } 7 \\
\text { pós-cirurgia. } \\
\text { O grupo LASER 5 J/ } \\
\text { cm }^{2} \text { mostrou mais alta } \\
\text { taxa de formação de } \\
\text { novos vasos quando } \\
\text { comparados aos } \\
\text { controles no dia } 21 . \\
\text { O grupo LASER } \\
20 \mathrm{~J} / \mathrm{cm}^{2} \text { foi similar ao } \\
\text { grupo controle. }\end{array}$ & NA & $\begin{array}{l}\text { A formação de } \\
\text { novos vasos foi } \\
\text { significativamente maior } \\
\text { nos grupos tratados } \\
\text { com LASER e LED, } \\
\text { com exceção do LASER } \\
20 \mathrm{~J} / \mathrm{cm}^{2} \text {. } \\
\text { A fluência de } 5 \mathrm{~J} / \mathrm{cm}^{2} \\
\text { estimulou a angiogênese } \\
\text { de forma mais intensa } \\
\text { do que os grupos com } \\
\text { fluência de } 20 \mathrm{~J} / \mathrm{cm}^{2} \text {. } \\
\text { O estudo não mostrou } \\
\text { diferença entre os } \\
\text { grupos de tratamento, } \\
\text { concluindo que, } \\
\text { provavelmente a } \\
\text { coerência não é fator } \\
\text { relevante para o efeito } \\
\text { angiogênico. }\end{array}$ \\
\hline Dall Agnol et. al. ${ }^{17}$ & $\begin{array}{l}\text { Comparar os efeitos } \\
\text { biomodulatórios da } \\
\text { luz coerente e não } \\
\text { coerente no processo } \\
\text { de reparo tecidual em } \\
\text { ratos diabéticos. }\end{array}$ & $\begin{array}{l}\text { Grupos tratados com } \\
\text { LED GaAlAs ( } \lambda 640 \mathrm{~nm} \text {; } \\
30 \mathrm{~mW}), 1 \text { dose logo } \\
\text { após procedimento } \\
\text { cirúrgico. } \\
\text { Grupos com LASER } \\
\text { InGaAIP ( } \lambda 660 \mathrm{~nm} ; \\
\left.6 \mathrm{~J} / \mathrm{cm}^{2}\right), 1 \text { dose, logo } \\
\text { após procedimento } \\
\text { cirúrgico. } \\
\text { A aplicação foi pontual } \\
\text { à distância de } 1 \mathrm{~cm} \text {. }\end{array}$ & $\begin{array}{l}\text { Redução significativa } \\
\text { do diâmetro da ferida } \\
\text { nos ratos diabéticos } 72 \mathrm{~h} \\
\text { após a lesão no grupo } \\
\text { tratado com LED vs. } \\
\text { controle. } \\
\text { Redução significativa do } \\
\text { diâmetro da ferida nos } \\
\text { ratos não diabéticos e } \\
\text { diabéticos } 7 \text { dias após } \\
\text { a lesão nos grupos } \\
\text { tratados com LED e } \\
\text { LASER vs. controle. }\end{array}$ & $\begin{array}{l}\text { A redução de células } \\
\text { inflamatórias e o } \\
\text { aumento no número } \\
\text { de vasos sanguíneos e } \\
\text { fibroblastos foi similar } \\
\text { nos grupos tratados } \\
\text { com LASER e LED } \\
\text { quando comparados } \\
\text { aos grupos não } \\
\text { tratados. }\end{array}$ & $\begin{array}{l}\text { LED e LASER } \\
\text { promoveram similar } \\
\text { aceleração do processo } \\
\text { de cicatrização cutânea } \\
\text { quando comparados aos } \\
\text { grupos controle. }\end{array}$ \\
\hline $\begin{array}{l}\text { Oliveira Sampaio } \\
\text { et al. }{ }^{19}\end{array}$ & $\begin{array}{l}\text { Avaliar os efeitos } \\
\text { do LASER e do LED } \\
\text { na proliferação de } \\
\text { fibroblastos em } \\
\text { feridas cutâneas em } \\
\text { ratos anêmicos com } \\
\text { deficiência de ferro. }\end{array}$ & $\begin{array}{l}\text { LASER de diodo } \\
\left.\text { (I660nm, } 4 \times 2.5 \mathrm{~J} / \mathrm{cm}^{2}\right) \\
\text { ou LED }(\lambda 700 \mathrm{~nm}) \\
\text { aplicados no dia } \\
\text { do procedimento } \\
\text { cirúrgico, } 48 \mathrm{~h}, 7,14 \\
\text { e } 21 \text { dias. } \\
\text { A aplicação foi pontual } \\
\text { em contato com a pele } \\
\text { (a ferida foi forrada } \\
\text { com papel filme). }\end{array}$ & NA & $\begin{array}{l}\text { Animais saudáveis } \\
\text { irradiados com LASER } \\
\text { e LED tiveram uma } \\
\text { elevada contagem de } \\
\text { fibroblastos. } \\
\text { Animais anêmicos } \\
\text { irradiados com } \\
\text { LED tiveram uma } \\
\text { elevada contagem de } \\
\text { fibroblastos. }\end{array}$ & $\begin{array}{l}\text { O estudo mostrou efeitos } \\
\text { biomodulatórios positivos } \\
\text { do LASER e LED em } \\
\text { ratos anêmicos. } \\
\text { A irradiação do LED } \\
\text { mostrou melhores } \\
\text { resultados em animais } \\
\text { anêmicos quando } \\
\text { comparados ao grupo } \\
\text { de LASER. }\end{array}$ \\
\hline De Souza et al. ${ }^{18}$ & $\begin{array}{l}\text { Comparar os efeitos } \\
\text { do LASER e do LED na } \\
\text { angiogênese de feridas } \\
\text { cutâneas em roedores. }\end{array}$ & $\begin{array}{l}\text { A fototerapia começou } \\
\text { imediatamente após } \\
\text { a cirurgia e se repetiu } \\
\text { todos os dias, durante } \\
7 \text { dias. } \\
\text { A aplicação foi pontual } \\
\text { em contato com a pele } \\
\text { (a ferida foi forrada } \\
\text { com papel filme). }\end{array}$ & NA & $\begin{array}{l}\text { A angiogênese foi } \\
\text { significativamente } \\
\text { aumentada nos grupos } \\
\text { de LED verde, LED } \\
\text { vermelho, LASER } \\
\text { infravermelho e LASER } \\
\text { vermelho. } \\
\text { Houve um significativo } \\
\text { aumento da } \\
\text { angiogênese no grupo } \\
\text { LED verde e vermelho } \\
\text { em relação ao grupo } \\
\text { LASER vermelho. }\end{array}$ & $\begin{array}{l}\text { LASER e LED são } \\
\text { capazes de estimular a } \\
\text { angiogênese em feridas } \\
\text { cutâneas. } \\
\text { A coerência não } \\
\text { foi decisiva para os } \\
\text { desfechos encontrados. }\end{array}$ \\
\hline Catão et al. ${ }^{21}$ & $\begin{array}{l}\text { Avaliar os efeitos do } \\
\text { LASER, do LED e da } \\
\text { terapia fotodinâmica } \\
\text { no processo de } \\
\text { cicatrização de } \\
\text { queimaduras. }\end{array}$ & $\begin{array}{l}5 \text { grupos: controle, } \\
\text { LASER } \lambda 660\left(10 \mathrm{~J} / \mathrm{cm}^{2}\right) \text {, } \\
\text { LASER } \lambda 780 \mathrm{~nm} \\
\left(60 \mathrm{~J} / \mathrm{cm}^{2}\right) \text {, terapia } \\
\text { fotodinâmica e } \\
\text { LED verde. } \\
\text { A irradiação foi } \\
\text { aplicada logo após } \\
\text { a queimadura, } \\
\text { diariamente. }\end{array}$ & $\begin{array}{l}\text { Até o } 14 \text { o dia, os } \\
\text { animais dos grupos de } \\
\text { tratamento apresentaram } \\
\text { um índice de retração } \\
\text { da ferida maior que o } \\
\text { grupo controle. } \\
\text { Aos } 21 \text { dias pós- } \\
\text { queimadura, } \\
\text { esta diferença foi } \\
\text { significativa nos grupos } \\
\text { LASER vermelho e } \\
\text { infravermelho vs demais } \\
\text { grupos. }\end{array}$ & $\begin{array}{l}\text { A produção e a } \\
\text { maturação de } \\
\text { fibroblastos foi } \\
\text { significativamente } \\
\text { aumentada em todos } \\
\text { os grupos tratados, } \\
\text { especialmente } \\
\text { nos grupos de } \\
\text { LASER vermelho e } \\
\text { infravermelho. }\end{array}$ & $\begin{array}{l}\text { LASER e LED podem } \\
\text { favorecer o processo } \\
\text { cicatricial após } \\
\text { queimaduras. } \\
\text { No entanto, os grupos } \\
\text { de LASER vermelho e } \\
\text { infravermelho mostraram } \\
\text { uma redução mais } \\
\text { significativa do tamanho } \\
\text { da ferida. }\end{array}$ \\
\hline
\end{tabular}


O primeiro estudo a comparar os efeitos biomodulatórios do LASER e do LED em feridas cutâneas foi desenvolvido pelo grupo de pesquisa da USP (Universidade de São Paulo ${ }^{20}$. Nesta investigação os autores compararam os efeitos fotoindutores do LASER $(\lambda 660 \mathrm{~nm})$ e do LED ( $\lambda 635 \mathrm{~nm}$ ) na formação vascular e no fechamento de feridas cutâneas. Os resultados mostraram que, independente da fluência utilizada (5 ou $20 \mathrm{~J} / \mathrm{cm}^{2}$ ), a terapia com LASER e LED estimulou significativamente a angiogênese no local da lesão. O aumento do leito vascular no local da ferida restaura as demandas nutricionais e de oxigênio para o tecido em formação, favorecendo a proliferação e a migração celular, assim como, a síntese de proteínas ${ }^{21,22}$.

Corazza et al. ${ }^{20}$ também observaram que o estímulo da angiogênese foi menos intenso nos grupos tratados com a fluência do LASER mais alta $\left(20 \mathrm{~J} / \mathrm{cm}^{2}\right)$. Em fototerapia a adequada determinação da densidade de energia é essencial para obtenção do efeito terapêutico. Estudos indicam que a bioestimulação do tecido cutâneo ocorra em densidades de energia entre 0,5 e $4 \mathrm{~J} / \mathrm{cm}^{2}$, contrariando a metodologia empregada nesta pesquisa ${ }^{23,24}$. Adicionalmente, os mesmos autores observaram que os resultados positivos da angiogênese foram similares nos grupos LASER e LED, sugerindo que a ação angiogênica da fototerapia esteve mais relacionada com o comprimento de onda e fluência de energia do que com a coerência da luz 15,16,25. Apesar do bom número amostral utilizado neste estudo ( $\mathrm{n}=24$ por grupo), a ausência de uma avaliação imuno-histoquímica específica, o grande desvio padrão das variáveis encontradas e a utilização de protocolos com densidade de energia $\left(20 \mathrm{~J} / \mathrm{cm}^{2}\right)$ pouco caracterizada na literatura limitam a análise dos desfechos.

No ano de 2009 o grupo da UNIVAP (Universidade do Vale do Paraíba) liderado por Dall Agnol et al. ${ }^{17}$ realizou um estudo similar, porém em ratos diabéticos. Na diabetes melittus os principais fatores que contribuem para a deficiência cicatricial são a diminuição da produção de fatores de crescimento e da angiogênese. Neste estudo os resultados mostraram que o diâmetro da ferida foi significativamente menor nos grupos tratados com LASER $(\lambda 660 \mathrm{~nm})$ e LED $(\lambda 640 \mathrm{~nm})$, principalmente nos animais diabéticos. Os autores concluíram que o LASER e o LED produziram efeitos similares de proliferação celular, sugerindo que a coerência da luz não é o principal requisito para estimular eventos bioquímicos. Além disso, também observou-se que, em apenas $72 \mathrm{~h}$ pós-lesão, os animais diabéticos tratados com LED apresentaram uma significativa redução no tamanho da ferida. Apesar das escassas evidências científicas, atualmente alguns autores tem postulado que a coerência da luz é perdida nas primeiras camadas da pele e que, portanto, esta característica não seria fundamental para produzir alterações celulares $^{15,16,25}$. No estudo de Dall Agnol et al. ${ }^{17}$, os autores hipotetizam que a eficácia aumentada no LED pode estar relacionada com o amplo espectro da fonte de luz não coerente, sugerindo que múltiplos comprimentos de onda podem estimular diferentes cromóforos e, assim, produzir diversas reações bioquímicas ao mesmo tempo. É importante ressaltar que a utilização de uma dose fototerapêutica única aplicada à distância de $1 \mathrm{~cm}$ do local da ferida limitam a análise dos resultados encontrados uma vez que há perda significativa de energia por reflexão.

Mais recentemente três artigos foram publicados sobre a temática desta revisão. Oliveira Sampaio et al. ${ }^{19}$ realizaram um estudo sobre os efeitos da fototerapia em ratos com deficiência de ferro. Os resultados desta investigação mostraram que o uso do LASER $(\lambda 660 \mathrm{~nm})$ ou do LED $(\lambda 700 \mathrm{~nm})$ estimulou a proliferação fibroblástica em ratos anêmicos e saudáveis. No entanto, animais anêmicos tratados com o LED vermelho obtiveram um resultado mais evidente do que o grupo tratado com LASER. Os autores sugerem que a deficiência de hemoglobina nos roedores anêmicos e o amplo espectro da luz do LED, constituíram fatores que facilitaram a absorção desta modalidade fototerapêutica. Barolet $^{10}$ (2008) e Schubert ${ }^{26}$ (2006) acrescentam ainda que, após a fotobioestimulação com uso do LED em fluências adequadas, ocorre uma rápida produção de ATP, estimulando a proliferação fibroblástica e a deposição de colágeno.

Similarmente, mas com animais sadios, De Souza et al. ${ }^{18}$ investigaram os efeitos do LASER e do LED na angiogênese após a indução de ferida cutânea. Utilizando diferentes comprimentos de onda, os autores mostraram que a fototerapia estimulou intensa angiogênese no local da ferida, corroborando estudos prévios que indicam que o LASER $^{27-29}$ e o LED ${ }^{15,16,25}$ nas faixas espectrais vermelha e infra-vermelha podem aumentar a vasodilatação e a microcirculação local, resultando no aumento do aporte de oxigênio e nutrientes na lesão cutânea. Além disso, também se observou que o LED no comprimento de onda verde foi capaz de estimular a angiogênese significativamente. Baseando-se em estudos prévios $^{30}$, os autores sugerem que, apesar dos relatos sobre a especificidade de absorção luminosa pelas biomoléculas presentes nos tecidos, verifica-se uma ampla janela biológica, permitindo a ação fotobiomoduladora em várias faixas do espectro com magnitudes diferentes.

Em 2015, Catão et al..$^{21}$ realizaram um estudo comparativo sobre o LASER, o LED e a terapia fotodinâmica (TFD) no processo de cicatrização de queimaduras. Os autores verificaram que até o 140 dia de aplicação da fototerapia, todos os grupos (LASER, LED e TFD) apresentaram um maior índice de retração da ferida quando comparados ao grupo controle. Aos 21 dias pós-queimadura, essa 
diferença foi observada apenas nos grupos de LASERs vermelho e infravermelho. Os pesquisadores sugerem que, nestes comprimentos de onda, a penetração do LASER seja maior, influenciando na maturação de fibroblastos para miofibroblastos já nas fases iniciais de cicatrização.

Cabe ressaltar que estudos como os de Corazza et al. ${ }^{20}$, De Souza et al. ${ }^{18} \mathrm{e}$ Catão et al. ${ }^{21}$, que utilizaram a irradiação do LASER e do LED em animais sem condições de risco para déficits de cicatrização são questionados na literatura. Smith ${ }^{31}$ (2005), pesquisador na área de fototerapia, sugere que em lesões agudas nos indivíduos sem condições patológicas associadas, os mecanismos fisiológicos trabalham de forma adequada e suficiente para reparar o tecido lesionado. Barolet $^{10}$ (2008) ainda acrescenta que células em homeostasia podem reagir pouco ou não reagir à fototerapia e, por isso, o seu efeito nem sempre é detectável. Já células em certas condições patológicas, tornam-se mais sensíveis à irradiação. Além disso, enfatiza-se que todos os estudos analisados nesta revisão apresentaram modelos animais de lesões cutâneas superficiais. É possível que estes resultados não possam ser reproduzidos em lesões cutâneas profundas, uma vez que luzes coerentes e não coerentes apresentam diferentes distribuições dentro do tecido ${ }^{31}$. Por fim, é importante acrescentar que o processo de reparo da pele humana apresenta mecanismos mais complexos de regeneração do que em animais de experimentação, sugerindo que estudos adicionais em seres humanos devam ser realizados para a padronização do uso clínico da fototerapia.

\section{CONSIDERAÇÕES FINAIS}

Os cinco artigos analisados nesta revisão mostraram resultados favoráveis ao uso de LASER e LED tanto em condições normais quanto em condições de risco para atraso na cicatrização cutânea. Os autores sugerem que os mecanismos de ação são similares em ambas as fototerapias LASER e LED e relacionam-se com a estimulação de fotorreceptores na cadeia respiratória mitocondrial, alterações nos níveis de ATP celular, liberação de fatores de crescimento e síntese de colágeno. No entanto, cabe ressaltar que, a carência de padronização metodológica dificulta conclusões precisas sobre os desfechos analisados nestes estudos comparativos. Além disso, é importante enfatizar que a terapia com LASER de baixa potência tem sido investigada há aproximadamente 20 anos e que, portanto, em função da significativa quantidade de publicações relevantes, ainda permanece como a abordagem fototerapêutica com embasamento científico mais consistente. Apesar destas limitações, os estudos apresentados nesta revisão mostram que a fototerapia de baixa potência possui promissora aplicabilidade clínica em processos cicatriciais cutâneos, sugerindo que este recurso possa se constituir em uma ferramenta fisioterapêutica valiosa, reduzindo complicações funcionais e estéticas. Certamente, o sucesso em roedores pode ser considerado o primeiro passo no desenvolvimento de uma abordagem terapêutica para um futuro uso clínico. No entanto, é evidente a necessidade de estudos adicionais em seres humanos a fim de definir estratégias seguras e efetivas para o uso da fototerapia em pacientes com dificuldades de cicatrização.

\section{REFERÊNCIAS}

1. Kede MPV. Dermatologia estética. São Paulo: Atheneu; 2009.

2. Flanagen M. Wound healing and skin integrity: principles and practice. Hoboken: Wiley-Blackwell; 2013.

3. Campos ACL, Borges-Branco A, Groth AK. Wound healing. Arq Bras Cir Dig. 2007;20(1):51-8. http://dx.doi.org/10.1590/S0102 67202007000100010

4. Brasil. Manual de condutas para úlceras neutróficas e traumáticas Brasília: Ministério da Saúde; 2002.

5. Singer AJ, Clark RA. Cutaneous wound healing. $N$ Engl J Med. 1999;341(10):738-46. http://dx.doi.org/10.1056/NEJM 199909023411006

6. Whinfield AL, Aitkenhead I. The light revival: does phototherapy promote wound healing? A review. Foot (Edinb). 2009;19(2): 117-24. http://dx.doi.org/10.1016/j.foot.2009.01.004

7. Peplow PV, Chung TY, Baxter GD. Laser photobiomodulation of wound healing: a review of experimental studies in mouse and rat animal models. Photomed Laser Surg. 2010;28(3):291-325. http:// dx.doi.org/10.1089/pho.2008.2446

8. Schreml S, Szeimies RM, Prantl L, Landthaler M, Babilas P. Wound healing in the 21st century. J Am Acad Dermatol. 2010;63(5): 866-81. http://dx.doi.org/10.1016/j.jaad.2009.10.048

9. Nouri K. Lasers in dermatology and medicine. Miami: Springer; 2012. http://dx.doi.org/10.1007/978-0-85729-281-0

10. Barolet D. Light-emitting diodes (LEDs) in dermatology. Semin Cutan Med Surg. 2008;27(4):227-38. http://dx.doi.org/10.1016/j. sder.2008.08.003

11. Roelandts R. The history of phototherapy: something new under the sun? J Am Acad Dermatol. 2002;46(6):926-30. http://dx.doi. org/10.1067/mjd.2002.121354

12. Mester E, Mester AF, Mester A. The biomedical effects of laser application. Lasers Surg Med. 1985;5(1):31-9. http://dx.doi. org/10.1002/lsm.1900050105

13. Avci P, Gupta A, Sadasivam M, Vecchio D, Pam Z, Pam N, Hamblin MR. Low-level laser (light) therapy (LLLT) in skin: stimulating, healing, restoring. Semin Cutan Med Surg. 2013;32(1):41-52.

14. Woodruff LD, Bounkeo JM, Brannon WM, Dawes KS, Barham CD, Waddell DL, Enwemeka CS. The efficacy of laser therapy in wound repair: a meta-analysis of the literature. Photomed Laser Surg. 2004;22(3):241-7. http://dx.doi.org/10.1089/1549541041438623 
15. Whelan HT, Smits RL Jr, Buchman EV, Whelan NT, Turner SG, Margolis DA, Cevenini V, Stinson H, Ignatius R, Martin T, Cwiklinski J, Philippi AF, Graf WR, Hodgson B, Gould L, Kane M, Chen G, Caviness J. Effect of NASA light-emitting diode irradiation on wound healing. J Clin Laser Med Surg. 2001;19(6):305-14. http://dx.doi. org/10.1089/104454701753342758

16. Whelan HT, Buchmann EV, Dhokalia A, Kane MP, Whelan NT, Wong-Riley MT, Eells JT, Gould LJ, Hammamieh R, Das R, Jett M. Effect of NASA light-emitting diode irradiation on wound healing. J Clin Laser Med Surg. 2003;21(2):67-74. http://dx.doi. org/10.1089/104454703765035484

17. Dall Agnol MA, Nicolau RA, De Lima CJ, Munin E. Comparative analysis of coherent light action (laser) versus non-coherent light (light-emitting diode) for tissue repair in diabetic rats. Lasers Med Sci. 2009;24(6):909-16. http://dx.doi.org/10.1007/s10103-009-0648-5

18. De Sousa AP, Paraguassú GM, Silveira NT, Souza J, Cangussú MC, Santos JN, Pinheiro AL. Laser and LED phototherapies on angiogenesis. Lasers Med Sci. 2013;28(3):981-7. http://dx.doi. org/10.1007/s10103-012-1187-z

19. Oliveira Sampaio SC, de C Monteiro JS, Cangussú MC, Pires Santos GM, dos Santos MA, dos Santos JN, Pinheiro AL. Effect of laser and LED phototherapies on the healing of cutaneous wound on healthy and iron-deficient Wistar rats and their impact on fibroblastic activity during wound healing. Lasers Med Sci. 2013;28(3):799-806. http:// dx.doi.org/10.1007/s10103-012-1161-9

20. Corazza AV, Jorge J, Kurachi C, Bagnato VS. Photobiomodulation on the angiogenesis of skin wounds in rats using different light sources. Photomed Laser Surg. 2007;25(2):102-6. http://dx.doi. org/10.1089/pho.2006.2011

21. de Vasconcelos Catão MH, Nonaka CF, de Albuquerque RL Jr, Bento PM, de Oliveira Costa R. Effects of red laser, infrared, photodynamic therapy, and green LED on the healing process of third-degree burns: clinical and histological study in rats. Lasers Med Sci. 2015;30(1):421-8. http://dx.doi.org/10.1007/s10103-014-1687-0
22. Enoch S, Leaper DJ. Basic science of wound healing. Surgery. 2008;26(2):37-42. http://dx.doi.org/10.1016/j.mpsur. 2007. 11.005

23. Huang YY, Sharma SK, Carroll J, Hamblin MR. Biphasic dose response in low level light therapy - an update. Dose Response. 2011; 9(4):602-18. http://dx.doi.org/10.2203/dose-response.11-009. Hamblin

24. Tuner JHL. Laser Therapy: Clinical practice and Scientific Background. Sweden: Prima Books; 2002.

25. Vinck EM, Cagnie BJ, Cornelissen MJ, Declercq HA, Cambier DC. Increased fibroblast proliferation induced by light emitting diode and low power laser irradiation. Lasers Med Sci. 2003;18(2):95-9. http://dx.doi.org/10.1007/s10103-003-0262-x

26. Schubert E. Light-Emitting Diodes. Cambridge: Cambridge University Press; 2006. http://dx.doi.org/10.1017/CBO9780511790546

27. Ihsan FR. Low-level laser therapy accelerates collateral circulation and enhances microcirculation. Photomed Laser Surg. 2005;23(3): 289-94. http://dx.doi.org/10.1089/pho.2005.23.289

28. Hussein AJ, Alfars AA, Falih MA, Hassan AN. Effects of a low level laser on the acceleration of wound healing in rabbits. N Am J Med Sci. 2011;3(4):193-7. http://dx.doi.org/10.4297/najms.2011.3193

29. Colombo F, Neto Ade A, Sousa AP, Marchionni AM, Pinheiro $A L$, Reis SR. Effect of low-level laser therapy on angiogenesis in wound healing: a immunohistochemical study in a rodent model. Braz Dent J. 2013;24(4):308-12. http://dx.doi.org/10.1590/01036440201301867

30. De Sousa AP, Santos JN, Dos Reis JA Jr, Ramos TA, de Souza J, Cangussú MC, Pinheiro AL.Effect of LED phototherapy of three distinct wavelengths on fibroblasts on wound healing: a histological study in a rodent model. Photomed Laser Surg. 2010;28(4):547-52. http://dx.doi.org/10.1089/pho.2009.2605

31. Smith KC. Laser (and LED) therapy is phototherapy. Photomed Laser Surg. 2005;23(1):78-80. http://dx.doi.org/10.1089/pho.2005.23.78 